\title{
Isomeric cross section study of neutron induced reactions on Ge isotopes
}

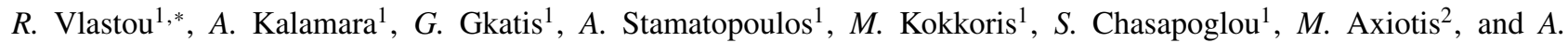 \\ Lagoyannis $^{2}$ \\ ${ }^{1}$ Department of Physics, National Technical University of Athens, Zografou campus, 15780 Athens, Greece. \\ ${ }^{2}$ Tandem Accelerator Laboratory, Institute of Nuclear Physics, N.C.S.R. Demokritos, Aghia Paraskevi, 15310 Athens, Greece.
}

\begin{abstract}
Cross sections for the ${ }^{70,76} \mathrm{Ge}(\mathrm{n}, 2 \mathrm{n}),{ }^{72,73} \mathrm{Ge}(\mathrm{n}, \mathrm{p})$ and ${ }^{72,74} \mathrm{Ge}(\mathrm{n}, \alpha)$ reactions have been measured at the 5.5 MV tandem T11/25 Accelerator Laboratory of NCSR Demokritos, using the activation technique. Neutron beams have been produced in the $\sim 16-20 \mathrm{MeV}$ energy region, by means of the ${ }^{3} \mathrm{H}(\mathrm{d}, \mathrm{n}){ }^{4} \mathrm{He}$ reaction. The maximum flux has been determined to be of the order of $10^{5} \mathrm{n} / \mathrm{cm}^{2} \mathrm{~s}$, while the flux variation of the neutron beam was monitored by using a $\mathrm{BF}_{3}$ detector. The cross section has been deduced with respect to the ${ }^{27} \mathrm{Al}(\mathrm{n}, \alpha){ }^{24} \mathrm{Na}$ and ${ }^{93} \mathrm{Nb}(\mathrm{n}, 2 \mathrm{n}){ }^{92 m} \mathrm{Nb}$ reference reactions. The contaminations from reactions induced on neighboring Ge isotopes and leading to the same residual nucleus, have been taken into account. After the end of the irradiations, the activity induced by the neutron beams at the targets and reference foils, has been measured by HPGe detectors. Statistical model calculations using the EMPIRE code were performed on the data measured in this work as well as on data reported in literature.
\end{abstract}

\section{Introduction}

Studies of excitation functions of neutron induced reactions are of considerable interest, not only for their importance to fundamental research in nuclear physics, but also for practical applications such as dosimetry and reactor technology [1-3]. Concerning Ge, besides its importance as a semi-conducting material, $(\mathrm{n}, 2 \mathrm{n})$ reactions on even-even Ge isotopes present high cross sections of hundreds of millibarns, thus increasing considerably the number of neutrons in neutron fields. In addition, $\mathrm{Ge}$ is expected to exhibit structure change and possible shape transition, having isotopes within the region of neutron number $\mathrm{N}=40$ and furthermore, some of the residual nuclei following $(\mathrm{n}, 2 \mathrm{n})$ and $(\mathrm{n}, \alpha)$ reactions on $\mathrm{Ge}$ isotopes, are produced in high spin isomeric states. The experimental determination of isomeric cross sections is of fundamental interest for studying the spin distribution of level density in the compound nucleus. Therefore, more experimental data are needed both for reliable practical applications as well as for testing theoretical calculations and improving the systematic development of model parameters.

Neutron-induced reactions on Ge isotopes have been investigated in the past by our group [4, 5], in the energy range of $\sim 8-11 \mathrm{MeV}$, by using the ${ }^{2} \mathrm{H}(\mathrm{d}, \mathrm{n})^{3} \mathrm{He}$ reaction. A survey of the available cross section data showed that reported measurements cover a wide energy range but with many discrepancies among them. In particular, extensive data exist at energies around $15 \mathrm{MeV}$, where the discrepancies are even higher, while only few data points exist in the higher energy regions. In view of these remarks, a continuation of this project has started for the measurement of

*e-mail: vlastou@central.ntua.gr neutron-induced threshold reaction cross sections on natural Ge, at energies from $\sim 15$ to $20 \mathrm{MeV}$, implementing the ${ }^{3} \mathrm{H}(\mathrm{d}, \mathrm{n}){ }^{4} \mathrm{He}$ reaction and the activation method. Furthermore, theoretical statistical model calculations have been performed, using the EMPIRE 3.2.2 code [6], and compared to all available experimental data.

\section{Experimental procedure}

\subsection{Irradiations}

The measurements were performed at the 5.5 MV tandem accelerator of NCSR Demokritos using quasimonoenergetic neutrons at a flux of the order of $\sim 10^{5}$ $\mathrm{n} /\left(\mathrm{cm}^{2} \mathrm{~s}\right)$ and energies 17.7 and $19.3 \mathrm{MeV}$, produced by the ${ }^{3} \mathrm{H}(\mathrm{d}, \mathrm{n})^{4} \mathrm{He}$ reaction by means of a Ti tritiated target, of $373 \mathrm{GBq}$ activity, on a Cu backing. The absolute flux of the beam was obtained with respect to the ${ }^{27} \mathrm{Al}(\mathrm{n}, \alpha)^{24} \mathrm{Na}$ and ${ }^{93} \mathrm{Nb}(\mathrm{n}, 2 \mathrm{n}){ }^{92 m} \mathrm{Nb}$ reference reactions, while its variation was monitored by a $\mathrm{BF}_{3}$ detector placed at a distance of $3 \mathrm{~m}$ from the neutron source. The spectra of the $\mathrm{BF}_{3}$ monitor were stored at regular time intervals $(\sim 200 \mathrm{~s})$ in a separate ADC during the irradiation process.

Samples of high purity Ge pellets doped with $6 \%$ high purity cellulose, were placed between $\mathrm{Al}$ and $\mathrm{Nb}$ reference foils and exposed to the neutron beam for about a twoday continuous irradiation. A gold foil was also used to cross check the experimental neutron flux, as well as the simulated one, via the ${ }^{197} \mathrm{Au}(\mathrm{n}, \gamma)$ reaction. The induced activity of product radionuclides in both target and reference foils was measured with HPGe detectors of $80 \%$ and $50 \%$ relative efficiency, properly shielded with lead blocks to reduce the contribution of the natural radioactivity. The efficiency of the detectors at the position of the 
activity measurements $(10 \mathrm{~cm})$ was determined via calibrated ${ }^{152} \mathrm{Eu},{ }^{133} \mathrm{Ba},{ }^{57} \mathrm{Co},{ }^{60} \mathrm{Co},{ }^{109} \mathrm{Cd},{ }^{54} \mathrm{Mn},{ }^{137} \mathrm{Cs}$ and ${ }^{214} \mathrm{Bi}$ point sources. The characteristic $\gamma$-rays were corrected for self-absorption in the target, summing effects of cascading transitions and counting geometry.

\subsection{Experimental results}

Among the possible reaction channels produced by the neutron irradiation of natural $\mathrm{Ge}$, only the ones which involve reasonable life time and gamma-ray de-excitation can be investigated by means of the activation technique. These are the ${ }^{72,73} \mathrm{Ge}(\mathrm{n}, \mathrm{p}){ }^{72,73} \mathrm{Ga},{ }^{72,74} \mathrm{Ge}(\mathrm{n}, \alpha)^{69,71} \mathrm{Zn}$ and ${ }^{70,76} \mathrm{Ge}(\mathrm{n}, 2 \mathrm{n}){ }^{69,75} \mathrm{Ge}$ reactions.

In the case of $(n, p)$ reaction channels on ${ }^{72,73} \mathrm{Ge},{ }^{72} \mathrm{Ga}$ decays with a lifetime of $14.1 \mathrm{~h}$ to ${ }^{72} \mathrm{Ge}$, whose deexcitation to its ground state proceeds through the characteristic $834.1,629.9$ and $2021.6 \mathrm{keV}$ transitions of $95.45 \%$, $26.87 \%$ and $26.13 \%$ intensity, respectively. ${ }^{73} \mathrm{Ga}$ decays with a lifetime of $4.86 \mathrm{~h}$ to ${ }^{73} \mathrm{Ge}$ whose de-excitation involves the $297.3 \mathrm{keV}$ gamma-ray of $79.8 \%$ intensity.

Concerning the $(n, \alpha)$ reactions on ${ }^{72,74} \mathrm{Ge}$, the residual nuclei ${ }^{69,71} \mathrm{Zn}$ constitute isomeric pairs with identical metastable and ground state spins. In more detail, ${ }^{69} \mathrm{Zn}$ is produced both in its $1 / 2^{-}$ground state $\left(\mathrm{T}_{1 / 2}=56.4 \mathrm{~min}\right)$ and its metastable $9 / 2^{+}\left(\mathrm{T}_{1 / 2}=13.76 \mathrm{~h}\right)$ state. The ground state of ${ }^{69} \mathrm{Zn}$ decays directly to the ground state of ${ }^{69} \mathrm{Ga}$, while the metastable state decays to the ground state of ${ }^{69} \mathrm{Zn}$ emitting the characteristic $438.6 \mathrm{keV}$ gamma-ray, with intensity $94.85 \%$, which can be used for the determination of the $\sigma_{m}$ cross section. ${ }^{71} \mathrm{Zn}$ is produced both in its $1 / 2^{-}$ground state $\left(T_{1 / 2}=2.45 \mathrm{~m}\right)$ and its metastable $9 / 2^{+}\left(\mathrm{T}_{1 / 2}=3.96 \mathrm{~h}\right)$ state. Due to the short lifetime of the ground state of ${ }^{71} \mathrm{Zn}$, only the activity of the metastable state could be measured via the activation technique. Thus, the $\sigma_{m}$ could be determined by analyzing the $386.3 \mathrm{keV}$ (with intensity $91.4 \%$ ) transition of ${ }^{71} \mathrm{Ga}$ which is fed by the de-excitation of the metastable state of ${ }^{71} \mathrm{Zn}$.

As for the $(\mathrm{n}, 2 \mathrm{n})$ reaction on ${ }^{70} \mathrm{Ge}$, the residual nucleus ${ }^{69} \mathrm{Ge}$ decays with a half-life of $39.05 \mathrm{~h}$ to ${ }^{69} \mathrm{Ga}$ which decays to its ground state through the characteristic gammarays $1106.7,574.1$ and $871.9 \mathrm{keV}$ with intensities $36 \%$, $13.3 \%$ and $11.9 \%$, respectively. The ${ }^{76} \mathrm{Ge}(\mathrm{n}, 2 \mathrm{n}){ }^{75} \mathrm{Ge}$ reaction leads to the formation of the unstable nucleus ${ }^{75} \mathrm{Ge}$ both in its $1 / 2^{-}$ground state $\left(\mathrm{T}_{1 / 2}=82.78 \mathrm{~m}\right)$ and its metastable $7 / 2^{+}\left(\mathrm{T}_{1 / 2}=47.7 \mathrm{~s}\right)$ state. The de-excitation of the metastable to the ground state in fraction $99.97 \%$, along with its short lifetime leads to the measurement of the total cross section $\sigma_{m+g}$ of the (n,2n) reaction via the $264.6 \mathrm{keV}$ (with intensity $11.4 \%$ ) characteristic transition of ${ }^{75}$ As.

The preliminary results for the cross section for all the above mentioned reaction channels, deduced from their characteristic gamma-rays, are presented in Fig. 1 in Section 3, along with data from literature and theoretical calculations. In the case of using several gamma-rays for the determination of the cross section of a reaction channel, the final value of the cross section is calculated as the weighted average of the individual values.

\subsection{Corrections for interfering reactions}

Since in this work germanium of natural isotopic composition was used as target material, some of the activation products may be formed via several interfering reactions. The residual nuclei ${ }^{72,73} \mathrm{Ga}$ from the ${ }^{72,73} \mathrm{Ge}(\mathrm{n}, \mathrm{p})$ reactions, could also be produced by the $(n, n p+d)$ processes on ${ }^{73} \mathrm{Ge}$ and ${ }^{74} \mathrm{Ge}$, respectively. In Ref. [7], contributions from these reactions have been estimated as 4 and $3 \mathrm{mb}$, respectively and were subtracted only for the higher energy measurements at $14.7 \mathrm{MeV}$. In the high energy region studied in this work however, the correction from the contaminant reactions are expected to be much higher. Indeed, by using the cross section of the $(n, n p+d)$ reaction on ${ }^{73} \mathrm{Ge}$ and ${ }^{74} \mathrm{Ge}$ from TENDL-2017 [8], corrections of the order of $24 \%$ and $74 \%$, respectively, were estimated for the neutron energy $17.7 \mathrm{MeV}$, while at $19.3 \mathrm{MeV}$ the corrections were $32 \%$ and $83 \%$, respectively for the ${ }^{72,73} \mathrm{Ge}(\mathrm{n}, \mathrm{p})$ reactions. A further correction is needed for the ${ }^{73} \mathrm{Ge}(\mathrm{n}, \mathrm{p})^{73} \mathrm{Ga}$ reaction arising from the ${ }^{76} \mathrm{Ge}(\mathrm{n}, \alpha)^{73} \mathrm{Zn}$ followed by $\beta$ decay to ${ }^{73} \mathrm{Ga}$, which was estimated to be $1 \%$ at 17.7 $\mathrm{MeV}$ and $0.5 \%$ at $19.3 \mathrm{MeV}$, using cross sections from [9]. Similarly, the contamination of the ${ }^{72} \mathrm{Ge}(\mathrm{n}, \alpha)$ reaction by the ${ }^{74} \mathrm{Ge}(\mathrm{n}, \mathrm{n} \alpha)^{69 m} \mathrm{Zn}$ one, was $27 \%$ and $35 \%$ at 17.7 and 19.3 MeV, respectively, as deduced by using the cross section from EAF-2010 [8]. Furthermore, the ${ }^{76} \mathrm{Ge}(\mathrm{n}, 2 \mathrm{n}){ }^{75} \mathrm{Ge}$ threshold reaction cross section is contaminated by the ${ }^{74} \mathrm{Ge}(\mathrm{n}, \gamma){ }^{75} \mathrm{Ge}$ one, which is activated by low energy parasitic neutrons. In order to estimate this neutron background contribution to the activation of ${ }^{75} \mathrm{Ge}$ and make the appropriate corrections, the reaction rate $\mathrm{RR}$ of the $(\mathrm{n}, \gamma)$ reaction was deduced by using the following expression:

$$
R R=\sum_{\Delta E} \sigma(E) \cdot \Phi\left(E_{i}\right)
$$

where $\sigma(E)$ is the excitation function of the ${ }^{74} \mathrm{Ge}(\mathrm{n}, \gamma)^{75} \mathrm{Ge}$ reaction taken from the ENDF/B-VII.1 library and $\Phi(E)$ the function of the neutron fluence estimated via Monte Carlo simulations using the NeuSDesc [11] and MCNP5 [12] codes. The neutron fluence, normalized to the experimental one from the reference reactions, is cut in energy slices $\Delta E$ starting from the threshold of each reaction up to the maximum beam energy. From the simulated RR, the expected number of counts were calculated and subtracted from the experimental number of counts for the $264.6 \mathrm{keV}$ characteristic $\gamma$-ray of the ${ }^{75} \mathrm{Ge}$ decay. In the absence of time-of-flight capabilities, the investigation of the neutron fluence energy dependence $\Phi(E)$ can only be achieved by means of Monte Carlo simulations taking into account all the details of the tritiated target, the experimental setup and the whole experimental area [13]. The simulations were executed for $10^{9}$ simulated particles, comparable to the number of neutrons used in the irradiations, for sufficient statistics. In order to test the reliability of the simulations, the methodology described above has been tested for the ${ }^{197} \mathrm{Au}(\mathrm{n}, \gamma)$ reaction and the simulated reaction rate has been compared with the experimental reaction rate deduced by the characteristic $411.8 \mathrm{keV} \gamma$-ray of this reaction. The simulated and experimental results were in 

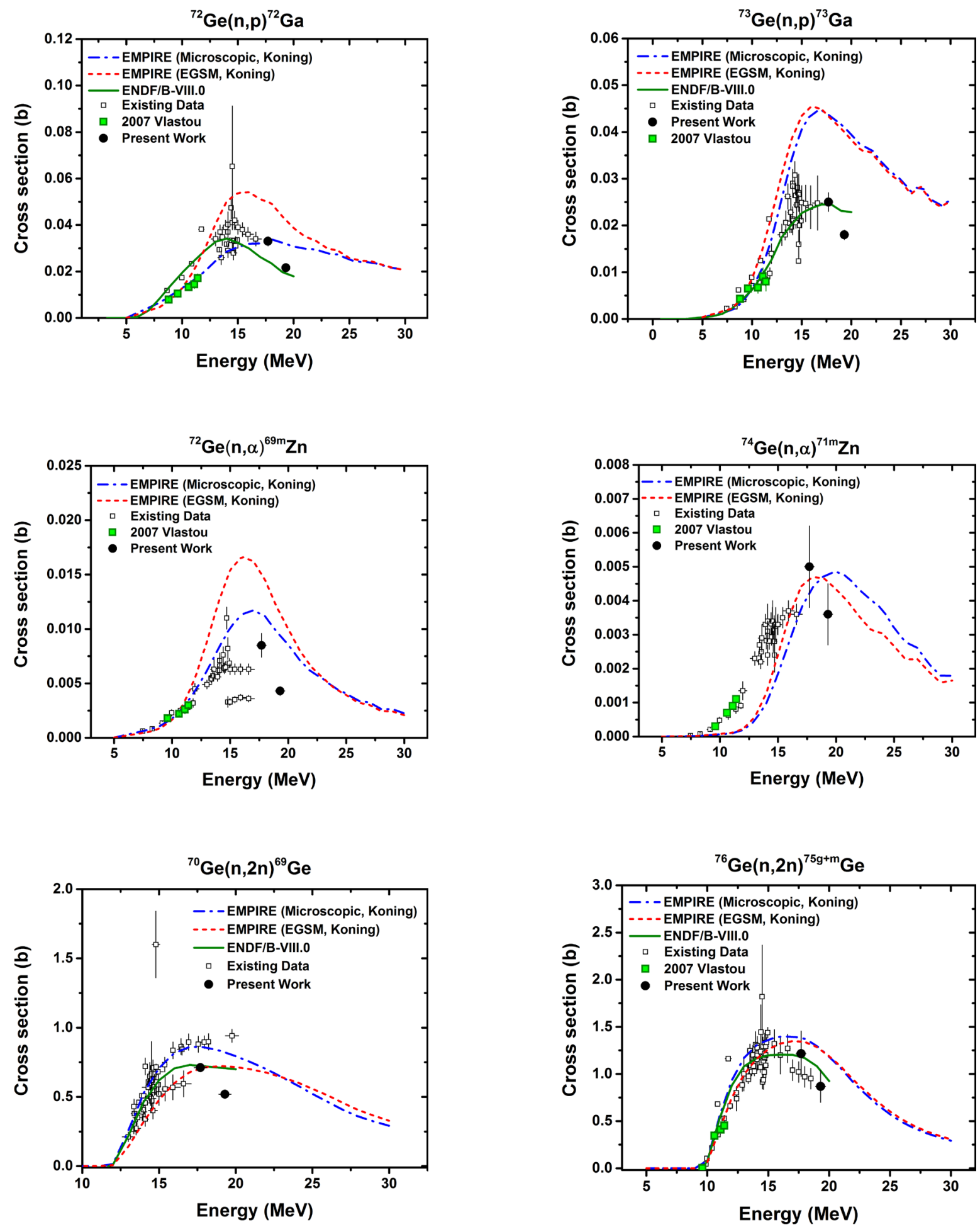

Figure 1: Experimental values of the present work at 17.7 and $19.3 \mathrm{MeV}$, along with EXFOR data from literature and theoretical calculations for the ${ }^{72,73} \mathrm{Ge}(\mathrm{n}, \mathrm{p}){ }^{72,73} \mathrm{Ga}$ (upper part), ${ }^{72,74} \mathrm{Ge}(\mathrm{n}, \alpha){ }^{69 m, 71 m} \mathrm{Zn}$ (middle part) and ${ }^{70,76} \mathrm{Ge}(\mathrm{n}, 2 \mathrm{n}){ }^{69,75 g+m} \mathrm{Ge}$ (lower part) reaction cross sections. The blue and red dashed lines are the results from the theoretical calculations using Microscopic HFB and EGSM level densities, respectively, while the green line corresponds to ENDF/B-VIII.0 evaluations.

good agreement, thus verifying the reliability of the simulations. The correction of ${ }^{76} \mathrm{Ge}(\mathrm{n}, 2 \mathrm{n})^{75} \mathrm{Ge}$ reaction cross section due to the interference of the ${ }^{74} \mathrm{Ge}(\mathrm{n}, \gamma)^{75} \mathrm{Ge}$ one, was estimated to be $0.8 \%$ at $17.7 \mathrm{MeV}$ and $19 \%$ at 19.3 $\mathrm{MeV}$.

\section{Theoretical calculations}

Excitation functions of neutron induced reactions on natural Ge present an interesting case since many isotopes are involved which allow an investigation of systematic trends in the data and in the energy region up to $20 \mathrm{MeV}$, many reaction channels are open, which may proceed via different reaction mechanisms such as $(n, 2 n)$, (n,p) and 
(n, $\alpha$ ), and therefore can be simultaneously studied. Furthermore, some of the residual nuclei following $(\mathrm{n}, 2 \mathrm{n})$ and $(\mathrm{n}, \alpha)$ reactions on $\mathrm{Ge}$ isotopes are produced in an isomeric state and are of high sensitivity for testing nuclear models. Thus, theoretical calculations based on the compound nucleus theory of Hauser-Feshbach, were performed in the energy range 5-30 MeV using the EMPIRE (3.2.2 version) code [6], for all reactions under study. The effect of different optical model potentials and level density models have been investigated and the ones presented below were found to better reproduce the data and were employed in the calculations. The level densities were described either by the Enhanced Generalized Superfluid Model (EGSM) [14] or by the RIPL-3 Microscopic Hartree-Fock-Bogoliubov (HFB) method [15]. Spherical optical model calculations were performed (DIRECT $=0$ ) with the optical model parameters for the outgoing neutrons and protons adopted by the data of Koning et al. [16], while for the alphas, the ones by Avrigeanu et al. [17] were used. Moreover, $\gamma$-ray strength functions were described via modified Lorentzians (MLO1) [18] with parameters available in RIPL-3 [19]. Furthermore, concerning the pre-equilibrium emission mechanism, the classical exciton model [20] was implemented via the PCROSS module [6] (PCROSS 2.2). The results are presented as red and blue dashed lines in Fig. 1, for the EGSM and Microscopic HFB level densities, respectively.

\section{Discussion and conclusions}

The preliminary experimental results of the ${ }^{72,73} \mathrm{Ge}(\mathrm{n}, \mathrm{p})^{72,73} \mathrm{Ga}, \quad{ }^{72,74} \mathrm{Ge}(\mathrm{n}, \alpha)^{69 m, 71 m} \mathrm{Zn}$ and ${ }^{70,76} \mathrm{Ge}(\mathrm{n}, 2 \mathrm{n})^{69,75 g+m} \mathrm{Ge}$ reactions at 17.7 and 19.3 $\mathrm{MeV}$ are presented in Fig. 1 along with data from literature. They are seen to follow the general trend of the existing experimental data, even though there are severe discrepancies among them, especially in the case of ${ }^{72} \mathrm{Ge}(\mathrm{n}, \alpha)^{69 m} \mathrm{Zn}$ and ${ }^{70} \mathrm{Ge}(\mathrm{n}, 2 \mathrm{n}){ }^{69} \mathrm{Ge}$. Concerning the statistical model calculations, the Microscopic HFB level density model exhibit a better agreement with the data of the various reaction channels compared to the EGSM, even for the reactions leading to metastable states. However, both theoretical calculations tend to overestimate all the high energy data. Furthermore, in the case of the (n,p) reaction on the even-odd isotope ${ }^{73} \mathrm{Ge}$, the theoretical results fail to reproduce the data. Therefore, further tests are needed before a firm conclusion is reached, mainly focused on the sensitivity of the calculations to variations of the level density parameter $\tilde{\alpha}$ and pre-equilibrium effects. In addition, the large discrepancies of the data do not allow to make possible validation of different model calculations. More accurate data are needed in the $15 \mathrm{C} 20 \mathrm{MeV}$ energy range in order to further investigate the contributions of the various reaction mechanisms and channels and test the reliability of the theoretical calculations. In fact, more measurements are planned to be performed by our group at $15-20 \mathrm{MeV}$ in the near future, in order to provide more experimental information which will help to resolve discrepancies among the existing experimental data and theoretical model calculations.

\section{Acknowledgements}

The authors would like to acknowledge the assistance of the Accelerator Staff at NCSR "Demokritos".

\section{References}

[1] P. Talou et al., Nucl. Sci. Eng. 155, 84 (2007).

[2] A. Fessler et al., Nucl. Sci. Eng. 134, 171 (2000).

[3] M. Avrigeanou et al., Phys. Rev. C 85, 044618 (2012).

[4] S.Galanopoulos et al., Nucl. Instr. Meth., B 261969 (2007).

[5] R.Vlastou et al., J. Radioanal. Nucl. Chem. 272, 219 (2007).

[6] M. Herman et al., Nucl. Data Sheets 108, 2655 (2007).

[7] I. Birn S.M.Qaim, Nucl. Sci. Eng. 116, 125 (1994).

[8] ENDF, https://doi.org/10.1016/j.nds. 2011.11.002

[9] A.J. Koning and D. Rochman, Nucl. Data Sheets 113 2841 (2012).

[10] J-Ch. Suble et al., The European Activation File: EAF-2010 neutron-induced cross Section LibraryCCFE-R (10) 05.

[11] E. Birgersson and G. Lovestam, JRC Science Hub Technical Report, Ref. No., 23794, 2009.

[12] X-5 Monte Carlo team, Volume I-III, LA-UR-031987, LA-CP-03 0245 and LA-CP-03-0284, April (2003).

[13] R.Vlastou et al., EPJ Web of Conferences 146, 11013 (2017).

[14] A. DArrigo et al., J. Phys. G 20, 365 (1994).

[15] S. Goriely, M. Samyn, and J. M. Pearson, Phys. Rev. C 75, 064312 (2007).

[16] A. J. Koning and J. P. Delaroch, Nucl. Phys. A 713, 231(2003).

[17] V. Avrigeanu et al., Phys. Rev. C 90, 044612 (2014).

[18] V. A. Plujko, Acta Phys. Pol. B 31, 435 (2000).

[19] R. Capote et al., https://www-nds.iaea.org/RIPL-3/, 2009.

[20] J. J. Griffin, Phys. Rev. Lett. 17, 478 (1966). 